\title{
Prevalence of mitochondrial gene mutations among hearing impaired patients
}

Shin-ichi Usami, Satoko Abe, Jiro Akita, Atsushi Namba, Hideichi Shinkawa, Masanori Ishii, Satoshi Iwasaki, Tomoyuki Hoshino, Juichi Ito, Katsumi Doi, Takeshi Kubo, Takashi Nakagawa, Sohtaro Komiyama, Tetsuya Tono, Shizuo Komune

\begin{abstract}
Department of Otorhinolaryngology, Hirosaki University School of Medicine, 5 Zaifu-cho, Hirosaki 036-8562, Japan

S Usami

S Abe

J Akita

A Namba

H Shinkawa
\end{abstract}

Department of Otorhinolaryngology, Tokyo Kosei Nenkin Hospital, Tokyo 162, Japan

M Ishii

Department of Otorhinolaryngology, Hamamatsu

University School of

Medicine, Hamamatsu

431-31, Japan

S Iwasaki

T Hoshino

Department of Otolaryngology, Otsu Red Cross Hospital, Otsu 520, Japan $\mathrm{J}$ Ito

Department of Otolaryngology, Osaka University Faculty of Medicine, Suita 565-0871, Japan K Doi

T Kubo

Department of Otorhinolaryngology, Faculty of Medicine,

Kyushu University, Fukuoka 812-8582, Japan

T Nakagawa

S Komiyama

Department of Otorhinolaryngology, Miyazaki Medical College, Miyazaki 889-16, Japan $\mathrm{T}$ Tono

S Komune

Correspondence to: Professor Usami, Department of Otorhinolaryngology, Shinshu University School of Medicine, 3-1-1 Asahi, Matsumoto 390-8621, Japan

Revised version received 16 August 1999 Accepted for publication 27 August 1999

\begin{abstract}
The frequency of three mitochondrial point mutations, $1555 \mathrm{~A} \rightarrow \mathrm{G}, 3243 \mathrm{~A} \rightarrow \mathrm{G}$, and $7445 A \rightarrow G$, known to be associated with hearing impairment, was examined using restriction fragment length polymorphism (RFLP) analysis in two Japanese groups: (1) 319 unrelated SNHL outpatients (including 21 with aminoglycoside antibiotic injection history), and (2) 140 cochlear implantation patients (including 22 with aminoglycoside induced hearing loss). Approximately $3 \%$ of the outpatients and $10 \%$ of the cochlear implantation patients had the $1555 \mathrm{~A} \rightarrow \mathrm{G}$ mutation. The frequency was higher in the patients with a history of aminoglycoside injection (outpatient group 33\%, cochlear implantation group 59\%). One outpatient $(0.314 \%)$ had the $3243 \mathrm{~A} \rightarrow \mathrm{G}$ mutation, but no outpatients had the $7445 \mathrm{~A} \rightarrow \mathrm{G}$ mutation and neither were found in the cochlear implantation group. The significance of the $1555 \mathrm{~A} \rightarrow \mathrm{G}$ mutation, the most prevalent mitochondrial mutation found in this study of a hearing impaired population in Japan, among subjects with specific backgrounds, such as aminoglycoside induced hearing loss, is evident.

(F Med Genet 2000;37:38-40)
\end{abstract}

Keywords: mitochondria; point mutation; hearing impairment; frequencies

Mitochondrial mutations have been shown to be responsible for syndromic as well as non-syndromic hearing impairment (see hereditary hearing loss home page, http://dnalabwww.uia.ac.be/dnalab/hhh/).

The $1555 \mathrm{~A} \rightarrow \mathrm{G}$ point mutation is associated with a susceptibility to aminoglycoside antibiotics. In addition, in recent studies, several hearing impaired patients bearing the $1555 \mathrm{~A} \rightarrow \mathrm{G}$ mutation who had no history of aminoglycoside injection were reported. ${ }^{12}$ It is likely that among the hearing impaired population there are a great number of subjects with the $1555 \mathrm{~A} \rightarrow \mathrm{G}$ mutation.

The $3243 \mathrm{~A} \rightarrow \mathrm{G}$ mutation has been reported in a high proportion of patients with clinical features of MELAS (mitochondrial myopathy, encephalopathy, lactic acidosis, and stroke-like episodes). ${ }^{3}$ This mutation was also found in patients with diabetes mellitus ${ }^{45}$ and hearing loss is known to be associated with this mutation. $^{6}$
The $7445 \mathrm{~A} \rightarrow \mathrm{G}$ mutation was reported to be associated with hearing loss in Scottish, New Zealand, and Japanese families. ${ }^{7-9}$ Palmoplanter keratoderma was also found in the New Zealand and Japanese families. On the basis of the growing genetic evidence stated above, attention should be paid to the genetic background of patients who visit clinics. However, to date, little is known about the frequency of this mutation among the hearing impaired population. In the present study, the frequency of three mitochondrial mutations among the hearing impaired population in Japan was evaluated.

Subjects and methods

The frequency of three mitochondrial point mutations, $\quad 1555 \mathrm{~A} \rightarrow \mathrm{G}, \quad 3243 \mathrm{~A} \rightarrow \mathrm{G}$, and $7445 \mathrm{~A} \rightarrow \mathrm{G}$, known to be associated with hearing impairment, was examined in two groups. All subjects gave informed consent to participation in the project. Group 1 consisted of 319 unrelated subjects with a mean age of 40.7 years (range 3-92) who visited outpatient clinics because of sensorineural hearing loss (SNHL). A history of aminoglycoside injection was present in 21 subjects and 21 were syndromic with diabetes mellitus. There were no other asssociated neurological signs. The mean age of onset was 32.0 (range 0-79). Twenty five subjects had a family history with one or more other family members having hearing loss (12 compatible with autosomal dominant, 11 with autosomal recessive, two with mitochondrial inheritance). The mean hearing level of these subjects was $58.6 \mathrm{~dB} ; 37.7 \%$ of the subjects had mild hearing loss (up to $49 \mathrm{~dB}$ ), $34.5 \%$ had moderate $(50-69 \mathrm{~dB}), 9.6 \%$ severe $(70-89 \mathrm{~dB})$, and $18.2 \%$ profound ( $90 \mathrm{~dB}$ and over). Group 2 consisted of 140 subjects who had received cochlear implantation because of profound hearing loss, including 22 subjects with aminoglycoside induced hearing loss.

The frequency of three mitochondrial mutations was examined using restriction fragment length polymorphism analysis (RFLP). The PCR products were also sequenced by means of an ABI sequencer 377XL (Perkin Elmer Co Ltd).

Screening for the $1555 \mathrm{~A} \rightarrow \mathrm{G}$ mitochondrial mutation has been previously described. ${ }^{10}{ }^{10}$ In brief, mitochondrial DNA was extracted from the blood and nucleotide 1252-1726 amplified by PCR. Digestion was performed with a restriction enzyme (Alw26I) and the digested sample then electrophoresed on an agarose gel. As seen in fig 1A, normal controls had two 

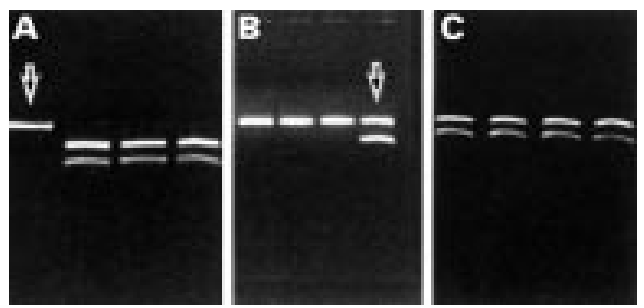

Figure 1 (A) $1555 A \rightarrow G$ mutation is indicated by an arrow. The restriction enzyme divides normal PCR products into two fragments. (B) $3243 A \rightarrow G$ mutation is indicated by an arrow. Mutant $m t D N A$ is divided into two fragments by the restriction enzyme. (C) Normal PCR products are divided into two fragments (mutant mtDNA was not available).

fragments, while in mutated DNA fragments loss of the Alw26I site, caused by the 1555 mutation, resulted in a single fragment. The 1555 mutation was confirmed by direct sequencing using an $\mathrm{ABI}$ sequencer $377 \mathrm{XL}$ (Perkin Elmer Co, Ltd).

To detect the $3243 \mathrm{~A} \rightarrow \mathrm{G}$ mutation, nucleotide 3160-3333 was amplified by PCR. Digestion was performed with a restriction enzyme (ApaI), and the digested sample then electrophoresed on an agarose gel. Mutated DNA fragments were digested into two fragments caused by the 3243 mutation (fig 1B). The 3243 mutation detected by RFLP analysis was confirmed by direct sequencing. All experiments included positive controls.

To detect the $7445 \mathrm{~A} \rightarrow \mathrm{G}$ mutation, nucleotide $7178-7840$ was amplified by PCR. Digestion was performed with a restriction enzyme $(X b a \mathrm{I})$, and the digested sample then electrophoresed on an agarose gel. Normal controls had two fragments (fig 1C), whereas mutated DNA fragments should be found as a single band.

\section{Results}

Table 1 summarises the results obtained in the present study.

The $1555 \mathrm{~A} \rightarrow \mathrm{G}$ mutation was found in (1) 11 out of the $319(3.45 \%)$ unrelated hearing impaired outpatients in group 1 ; (2) seven out of $21(33.3 \%)$ group 1 subjects with a history of aminoglycoside exposure; (3) 14 out of the $140(10 \%)$ cochlear implantation patients in group 2; and (4) 13 out of 22 (59\%) group 2 subjects with aminoglycoside induced hearing loss. In the subjects in group 1 with the $1555 \mathrm{~A} \rightarrow \mathrm{G}$ mutation, the mean age was 33.4 years (range 8-59) and the mean age of onset was 17.75 years (range 3-57). Two of these subjects compatible with mitochondrial inheritance had a $1555 \mathrm{~A} \rightarrow \mathrm{G}$ mutation. The mean hearing level of the patients with the

Table 1 Frequency of mitochondrial mutations in the hearing impaired patients

\begin{tabular}{|c|c|c|c|c|c|}
\hline \multirow[b]{2}{*}{ Mutation } & \multicolumn{3}{|l|}{ Group 1} & \multicolumn{2}{|l|}{ Group 2} \\
\hline & SNHL & $(S N H L+A G)$ & $(S N H L+D M)$ & $C I$ & $(C I+A G)$ \\
\hline $1555 \mathrm{~A} \rightarrow \mathrm{G}$ & $11 / 319$ & $(7 / 21)$ & & $14 / 140$ & $(13 / 22)$ \\
\hline $3243 \mathrm{~A} \rightarrow \mathrm{G}$ & $1 / 319$ & & $(1 / 21)$ & $0 / 140$ & \\
\hline $7445 \mathrm{~A} \rightarrow \mathrm{G}$ & $0 / 319$ & & & $0 / 140$ & \\
\hline
\end{tabular}

SNHL: patients who visited outpatient clinics for sensorineural hearing loss. CI: patients who received cochlear implant.

+AG: patients with history of aminoglycoside injection.

DM: patients with diabetes mellitus.
$1555 \mathrm{~A} \rightarrow \mathrm{G}$ mutation was $52.5 \mathrm{~dB} ; 44.4 \%$ of the subjects had mild hearing loss (up to 49 $\mathrm{dB}), 22.2 \%$ had moderate $(50-69 \mathrm{~dB}), 5.6 \%$ severe $(70-89 \mathrm{~dB})$, and $27.8 \%$ profound $(90$ $\mathrm{dB}$ and over).

The $3243 \mathrm{~A} \rightarrow \mathrm{G}$ mutation was found in one patient in group 1, a frequency of $0.314 \%$ $(1 / 319)$ in unrelated hearing impaired outpatients, and $4.76 \%(1 / 21)$ in the patients with diabetes mellitus. This patient had been referred to our clinic because of bilateral hearing loss associated with diabetes.

The $7445 \mathrm{~A} \rightarrow \mathrm{G}$ mutation was not found in the present subjects.

\section{Discussion}

The data presented suggest that the $1555 \mathrm{~A} \rightarrow \mathrm{G}$ mutation is one of the most important mutations among the hearing impaired population in Japan, and approximately $3 \%$ of patients with SNHL possess this mutation. The significance of this mutation among the subjects with the specific background of aminoglycoside induced hearing loss is evident. Therefore, it should be noted that this mutation is the most probable candidate for the gene responsible for hearing impairment in patients with aminoglycoside exposure. Two subjects had a family history on the maternal side, suggesting that family history should also be considered, but other factors, such as age or severity of hearing loss, are not significant criteria for the selection of patients to undergo mtDNA analysis. Although the mean age of onset in patients with the $1555 \mathrm{~A} \rightarrow \mathrm{G}$ mutation is younger than in those patients without the mutation, this is accounted for by the early age of aminoglycoside exposure.

This study showed a high frequency of this mutation among the deaf population with a history of aminoglycoside injection. It should be noted that profound hearing loss is possibly preventable and care must be taken when using aminoglycoside antibiotics. A rapid screening method as well as careful counselling ${ }^{11}$ should be widely instituted to prevent aminoglycoside induced hearing loss. Aminoglycoside antibiotics are still in use, especially in parts of Asia. The most common cause of hearing loss in China is aminoglycoside induced SNHL. ${ }^{12}$ The newly developed aminoglycoside antibiotics, which have less serious side effects, should also be used with caution as two subjects with the $1555 \mathrm{~A} \rightarrow \mathrm{G}$ mitochondrial mutation were found to have experienced hearing loss after short term, therapeutic dosage exposure to them. ${ }^{10}$ Genetic backgrounds should be adequately checked before aminoglycoside antibiotics are used in patients from high risk populations.

The $1555 \mathrm{~A} \rightarrow \mathrm{G}$ mutation was first reported in African and Asian populations, raising the possibility of a common ancestor for these groups. However, the results of recent phylogenetic analysis of 10 independent African and Asian families with the $1555 \mathrm{~A} \rightarrow \mathrm{G}$ mutation ${ }^{13}$ and 13 families in Japan $^{14}$ suggested that the $1555 \mathrm{~A} \rightarrow \mathrm{G}$ mitochondrial mutation has multiple origins. In addition, this mutation has been recently found in Greek, English/Irish, Italian, 
Mexican, Puerto Rican, and Vietnamese, ${ }^{15}$ and Spanish and Cuban ${ }^{216}$ populations. The $1555 \mathrm{~A} \rightarrow \mathrm{G}$ mitochondrial mutation was also commonly found in Spanish hereditary hearing loss families $(27.1 \%$ of all families and $55.9 \%$ of families with progressive deafness). This indicates that high risk populations may be found world wide.

It should be noted that a high frequency of the $1555 \mathrm{~A} \rightarrow \mathrm{G}$ mutation has been found in the profound hearing impaired populations. Of patients who received cochlear implantation because of aminoglycoside induced hearing loss, $57 \%$ had this mutation. We have reported good results with cochlear implantation in one patient with aminoglycoside induced hearing loss and the $1555 \mathrm{~A} \rightarrow \mathrm{G}$ mutation. ${ }^{17}$

A series of reports has suggested that this mutation also causes hearing loss even without aminoglycoside injection, ${ }^{12}$ although the hearing impairment in such cases is usually milder. ${ }^{1}$ In the present study, four out of 11 subjects (group 1) and one out of 14 subjects (group 2) bearing the $1555 \mathrm{~A} \rightarrow \mathrm{G}$ mutation did not have any history of aminoglycoside injection. Therefore, this mutation is an important disease causing mutation for non-syndromic hearing loss unrelated to aminoglycoside antibiotics.

Concerning the frequency of the 3243 mutation, it has been found in approximately $1 \%$ of patients with diabetes mellitus. ${ }^{6}$ According to Kadowaki et $a l,{ }^{6} 61 \%$ of the diabetes patients with the $3243 \mathrm{~A} \rightarrow \mathrm{G}$ mutation have hearing loss. The present study provided the frequency $(0.314 \%)$ of the $3243 \mathrm{~A} \rightarrow \mathrm{G}$ mutation in the hearing impaired population. Furthermore, this mutation was found more frequently $(4.76 \%)$ in the patients who had diabetes mellitus. This mutation should be considered as a cause of hearing loss in the patients who have diabetes.

The absence of the $7445 \mathrm{~A} \rightarrow \mathrm{G}$ mutation suggested that this mutation is not a major cause of hearing loss in the Japanese population.

In conclusion, genetic testing showed the frequency of three mitochondrial mutations among the hearing impaired population in Japan. The high frequency of the $1555 \mathrm{~A} \rightarrow \mathrm{G}$ mutation in hearing impaired populations, especially in those with specific backgrounds, indicates that genetic backgrounds should be adequately checked in these patients.
We thank all the families that participated in the present project. We would also like to thank Y Kon, Tsuyoshi Sakai, and Kyokugen Loo for technical assistance, and A C AppleMathews for help in preparing the manuscript. This study was supported by Health Sciences Research Grant (Research on Eye and Ear Science, Immunology, Allergy and Organ Transplantation) from the the Acute Profound Deafness Research Committee of the Ministry of Health and Welfare of Japan.

1 Usami S, Abe S, Kasai M, et al. Genetic and clinical features of sensorineural hearing loss associated with the 1555 mitochondrial mutation. Laryngoscope 1997;107:483-90.

2 Estivill X, Govea N, Barceló A, et al. Familial progressive sensorineural deafness is mainly due to the mtDNA A1555G mutation and is enhanced by treatment with aminoglycosides. Am f Hum Genet 1998;62:27-35.

3 Goto Y, Nonaka I, Horai S. A mutation in the tRNA(Leu)(UUR) gene associated with the MELAS subgroup of mitochondrial encephalomyopathies. Nature 1990;348: 651-3.

4 van den Ouweland JM, Lemkes HH, Ruitenbeek W, et al. Mutation in mitochondrial tRNA ${ }^{\text {Leu(UUR) }}$ gene in a large pedigree with maternally transmitted type II diabetes mellitus and deafness. Nat Genet 1992;1:368-71.

5 Reardon W, Ross RI, Sweeney MG, et al. Diabetes mellitus associated with pathogenic point mutation in mitochondrial DNA. Lancet 1992;340:1376-9.

6 Kadowaki T, Kadowaki H, Mori Y, et al. A subtype of diabetes mellitus associated with a mutation of mitochondrial DNA. N Engl f Med 1994;330:962-8.

7 Reid FM, Vernham GA, Jacobs HT. A novel mitochondrial point mutation in a maternal pedigree with sensorineural deafness. Hum Mutat 1994;3:243-7.

8 Fischel-Ghodsian N, Prezant TR, Fournier P, Stewart IA, Maw M. Mitochondrial mutation associated with nonsyndromic deafness. Am 7 Otolaryngol 1995;16:403-8.

9 Sevior KB, Hatamochi A, Stewart IA, et al. Mitochondrial A7445G mutation in two pedigrees with palmoplantar keratoderma and deafness. Am F Med Genet 1998;75:17985 .

10 Usami S, Abe S, Tono T, Komune S, Kimberling WJ, Shinkawa $H$. Isepamicin sulfate-induced sensorineural hearing loss in patients with the $1555 \mathrm{~A} \rightarrow \mathrm{G}$ mitochondrial mutation. ORL 1998;60:164-9.

11 Usami S, Abe S, Shinkawa H, Inoue Y, Yamaguchi T. Rapid mass screening method and counseling for the $1555 \mathrm{~A} \rightarrow \mathrm{G}$ mitochondrial mutation. f Hum Genet 1999;44:304-7.

12 Fu DM. Survey of 1583 deaf mutes. Qinghai Med f 1985;1: 22-3.

13 Hutchin TP, Cortopassi GA. Multiple origins of a mitochondrial mutation conferring deafness. Genetics 1997;145:771-6

14 Abe S, Usami S, Shinkawa H, et al. Phylogenetic analysis of mitochondrial DNA in Japanese pedigrees of sensorineural hearing loss associated with the A1555G mutation. Eur $\mathcal{F}$ Hum Genet 1998;6:563-9.

15 Fischel-Ghodsian N, Prezant TR, Chaltraw WE, et al. Mitochondrial gene mutation is a significant predisposing factor in aminoglycoside ototoxicity. Am f Otolaryngol 1997;18: 173-8.

16 Sarduy M, del Castillo I, Villamar M, et al. Genetic study of mitochondrially-inherited sensorineural deafness in eight large families from Spain and Cuba. 2nd Workshop of European Work Group on Genetics of Hearing Impairment, Milan, pean Work

17 Tono T, Ushisako Y, Kiyomizu K, et al. Cochlear implantation in a patient with profound hearing loss with the 1555A $\rightarrow \mathrm{G}$ mitochondrial mutation. Am f Otol 1998;19: $754-7$. 